\title{
Occupational Contact Dermatitis
}

\author{
Denis Sasseville, MD, FRCPC
}

\begin{abstract}
Occupational contact dermatitis accounts for $\mathbf{9 0} \%$ of all cases of work-related cutaneous disorders. It can be divided into irritant contact dermatitis, which occurs in $\mathbf{8 0} \%$ of cases, and allergic contact dermatitis. In most cases, both types will present as eczematous lesions on exposed parts of the body, notably the hands. Accurate diagnosis relies on meticulous history taking, thorough physical examination, careful reading of Material Safety Data Sheets to distinguish between irritants and allergens, and comprehensive patch testing to confirm or rule out allergic sensitization. This article reviews the pathogenesis and clinical manifestations of occupational contact dermatitis and provides diagnostic guidelines and a rational approach to management of these often frustrating cases.
\end{abstract}

Key words: allergic contact dermatitis, irritant contact dermatitis, occupational, work related

$T$ he skin is our primary interface with the external environment and, in general, performs quite efficiently as a barrier against noxious chemicals or living organisms. The range of human activities is extremely diversified, and numerous occupations can lead to breakdown of the epidermal barrier, with subsequent development of work-related dermatoses.

Exposure in the workplace is responsible for a wide range of cutaneous problems, as summarized in Table 1. Contact dermatitis, however, accounts for $90 \%$ of all cases of occupational dermatoses. ${ }^{1,2}$ The true prevalence of occupational contact dermatitis is unknown as many workers never report minor ailments. Those with more severe conditions are initially managed, and sometimes mismanaged, by primary care physicians, and some end up referred to dermatologists and allergists. It is important that the physician who takes charge of these patients knows how to recognize, investigate, and treat this disabling condition. The present article reviews the types, etiology, and clinical presentation of occupational contact dermatitis and provides the reader with a rational approach to this often vexing problem.

Denis Sasseville: Division of Dermatology, McGill University Health Centre, Montreal, QC.

Correspondence to: Denis Sasseville, MD, FRCPC, Division of Dermatology, McGill University Health Centre, Royal Victoria Hospital, Room A 4.17, 687 Pine Avenue West, Montreal, QC H3A 1A1; e-mail: denis.sasseville@mcgill.ca.

DOI $10.2310 / 7480.2008 .00010$

\section{Irritant Contact Dermatitis}

Irritant contact dermatitis (ICD) is the most common type of occupational skin disorder, traditionally held accountable for approximately $80 \%$ of all cases (Table 2). It is caused by the direct cytotoxic action of the offending agent on the cells of the epidermis and dermis. Visible skin changes are the result of alterations in the epidermal barrier, cellular destruction, transepidermal water loss, and inflammation secondary to non-immunologic release of vasoactive peptides and proinflammatory cytokines.

Irritants are mostly chemicals, in solid, liquid, or gaseous phase, but also include mineral or vegetal particles that abrade or get imbedded in the skin. Immediate irritants are corrosive substances that produce chemical burns within minutes to hours of a single exposure. Cumulative irritants are weaker substances such as detergents or solvents that require repeated application to exert their noxious effects (Table 3). The threshold for irritation varies from one individual to another, and a single individual may experience, over a period of time, hardening or loss of tolerance. However, with sufficient exposure and high enough concentration of the irritant, everyone is prone to the development of ICD. Although itch is a frequent complaint, the main symptoms are pain or a burning sensation, and the dermatitis presents as subacute to chronic eczema. ${ }^{3}$

\section{Allergic Contact Dermatitis}

A prototype of cell-mediated immune reaction, allergic contact dermatitis (ACD) is responsible for $20 \%$ of cases of occupational dermatitis. It occurs in a minority of 
Table 1. Classification of Occupational Dermatoses

\begin{tabular}{|c|c|}
\hline Type of Dermatosis & Example or Cause \\
\hline \multicolumn{2}{|l|}{ Contact dermatitis } \\
\hline Irritant & Solvents, detergents \\
\hline Allergic & $\begin{array}{l}\text { p-Phenylenediamine in } \\
\text { hairdressers }\end{array}$ \\
\hline \multicolumn{2}{|l|}{ Contact urticaria } \\
\hline Immunologic & Natural rubber latex, crabmeat \\
\hline Non-immunologic & $\begin{array}{l}\text { Ammonium persulfate } \\
\text { (hairdressers) }\end{array}$ \\
\hline \multicolumn{2}{|l|}{ Infections } \\
\hline Bacterial & Erysipelothrix in fishmongers \\
\hline Fungal & Sporotrichosis in gardeners \\
\hline Viral & Warts in butchers \\
\hline Parasitic & Cheyletiellosis in veterinarians \\
\hline \multicolumn{2}{|l|}{ Hair follicle disorders } \\
\hline Folliculitis & Motor oil in mechanics \\
\hline Chloracne & Polychlorinated biphenyls \\
\hline \multicolumn{2}{|l|}{ Pigmentation disorders } \\
\hline $\begin{array}{l}\text { Post-inflammatory } \\
\text { hyperpigmentation }\end{array}$ & Phytophotodermatitis \\
\hline Acquired leukoderma & Hydroquinones in rubber/plastics \\
\hline \multicolumn{2}{|l|}{ Neoplasms } \\
\hline Granulomas & Foreign bodies, beryllium \\
\hline \multirow{2}{*}{$\begin{array}{l}\text { Benign tumours or } \\
\text { carcinomas }\end{array}$} & Anthracene in soot or petroleum \\
\hline & Ionizing or ultraviolet radiation \\
\hline \multicolumn{2}{|l|}{ Miscellaneous } \\
\hline Scleroderma & Vinyl chloride \\
\hline Raynaud phenomenon & Vibrating tools \\
\hline Telangiectasias & Aluminum smelter workers \\
\hline
\end{tabular}

individuals and is caused by chemical or biological agents that are otherwise innocuous to the vast majority of people. The sequence of events that generate visible dermatitis is a biphasic process.
Table 3. Common Occupational Cutaneous Irritants

Acids and alkalis

Solvents

Aliphatic: petroleum, kerosene, gasoline

Aromatic: benzene, toluene, xylene

Halogenated: chloroform, trichloroethylene, methylchloride

Miscellaneous: water, alcohols, ketones, glycols, turpentine

Soaps and detergents

Plastics and resins

Epoxy, phenolic and acrylic monomers

Amine catalysts

Styrene, benzoyl peroxide

Metal salts

Nickel, chromium, cobalt, platinum, arsenic

Plants

Bristles, thorns

Calcium oxalate: dieffenbachia, philodendron, daffodil, agave

Phototoxic psoralens: Apiaceae, Rutaceae

Particles

Sand, sawdust, fiberglass, metal filings, etc.

\section{Sensitization Phase}

Most allergens are lipophilic and small $(<500$ D) molecules capable of penetrating the stratum corneum and reaching antigen-presenting cells (APCs) in the epidermis (Langerhans cells) or dermis (dermal dendritic cells). These chemicals are incomplete antigens, or haptens, that must be captured by APCs, internalized, bound to proteins of the major histocompatibility complex, and reexpressed at the cell surface to become complete antigens. APCs migrate to local lymph nodes, where they present the newly formed allergens to naive $\mathrm{T}$ cells. These lymphocytes subsequently undergo clonal proliferation and differentiation into CD4 and CD8 effector, suppressor, and memory cells that are liberated in the bloodstream and home for the skin. This process

Table 2. Distinguishing Features of Irritant and Allergic Contact Dermatitis

\begin{tabular}{lll}
\hline Feature & \multicolumn{1}{c}{ Irritant Contact Dermatitis } & \multicolumn{1}{c}{ Allergic Contact Dermatitis } \\
\hline Pathogenesis & Direct cytotoxic effect & T cell-mediated immune reaction \\
Affected individuals & Everyone & A minority of individuals \\
Onset & Immediate (chemical burns) & 12-48 h in previously sensitized individuals \\
& After repeated exposure to weak irritants & \\
Signs & Subacute or chronic eczema with & Acute to subacute eczema with vesiculation \\
& desquamation, fissures & \\
Symptoms & Pain or burning sensation & Pruritus \\
Concentration of contactant & High & Low \\
Investigation & None & Patch or prick tests \\
\hline
\end{tabular}


takes place over 10 to 15 days and rarely gives rise to visible skin lesions.

\section{Elicitation Phase}

Reexposure to the allergen results in priming of previously sensitized $\mathrm{T}$ cells to produce interleukin (IL)-1, IL-2, and interferon- $\gamma$. These lymphokines induce proliferation of cytotoxic $\mathrm{T}$ cells and recruitment of macrophages. Within 8 to 48 hours, these effector cells and their proinflammatory cytokines will attack the epidermis and generate the clinical picture of dermatitis. Untreated, this process may go on for days or weeks, until suppressor cells that secrete mainly IL-4 and IL-10 take over and inhibit the reaction. ${ }^{4}$

Although clinical signs of ICD and ACD often overlap and cannot always be distinguished, ACD tends to manifest as acute to subacute dermatitis, with pruritus as its cardinal symptom. In sensitized individuals, the concentration of the allergen needed to induce lesions may be extremely low (see Table 2).

\section{Clinical Presentation}

Occupational contact dermatitis presents as eczema in $90 \%$ of cases. Acute lesions begin as pruritic erythematous and edematous, urticarial-looking plaques that become rapidly studded with vesicles and sometimes tense bullae. A clear serous exudate escapes when these blisters rupture. Erythema and edema are still present in the subacute stages, but vesiculation becomes less visible, replaced by erosions, oozing, crusting, and desquamation. In longstanding, chronic cases, the skin appears dry and rough, fissured, grayish, and thickened with increased skin lines, a process called lichenification.

In rare cases, the morphology of the eruption may be different. Contact urticaria, as exemplified by natural rubber latex hypersensitivity, is an immediate, immunoglobulin E-mediated reaction characterized by transient edematous wheals without epidermal changes. Protein contact dermatitis, sometimes seen in food handlers, bakers, and veterinarians, begins as an urticarial reaction and is followed in a few days by an eczematous phase. Hypersensitivity reactions to strong allergens such as poison ivy or exotic woods sometimes present as widespread erythema multiforme with target lesions. Exposure to colour film developer is known to induce lichen planuslike lesions, characterized by flat-topped, slightly scaly, violaceous, and polygonal papules that coalesce to form irregular plaques.
The hands are the primary site of involvement in $80 \%$ of cases of occupational dermatitis, followed by the wrists and forearms. ICD from liquids such as water and detergents affects the fingertips and the web spaces. Allergy to rubber chemicals in gloves presents as dermatitis of the dorsal hand, whereas the palm is more often affected by allergy to solid objects. The hands may transfer irritants and allergens to distant sites such as the face. Airborne exposure to particulate matter, such as sawdust and fibreglass, or the smoke, fumes, and vapours of volatile chemicals causes lesions on the face, upper eyelids, ears, scalp, neck, and other exposed areas, sometimes infiltrating clothes.

In general, involvement of covered areas, genitals, or feet is not suggestive of occupational origin, but exceptions do occur: work clothes may become saturated with liquids, oil, or grease, giving rise to lesions on the legs, thighs, and abdomen, whereas lesions on the feet may signify allergy to workboots. The very fine sawdust generated by sanding exotic woods is very pervasive and can cause lesions that are more prominent in areas of friction from clothes such as body folds and genitals.

At times, the pattern of the dermatitis suggests the cause. Linear streaks of papules and vesicles are characteristic of phytodermatitis, whereas photocontact dermatitis will affect areas exposed to light, sparing the upper eyelids and submental and retroauricular areas.

ICD tends to remain localized to the area of contact, whereas ACD has a propensity to spread to more distant sites, either by a process known as autoeczematization or through the phenomenon of systemic contact dermatitis. The latter occurs when an individual previously sensitized by cutaneous exposure is exposed to the allergen orally or parenterally: a worker sensitized to ethylenediamine present in cutting oils could develop a generalized dermatitis on administration of structurally related medications such as hydroxyzine or aminophylline.

\section{The Offenders}

\section{Irritants}

The vast majority of irritants are chemicals. Strong acids and alkalis, concentrated solutions of sodium hypochlorite, isothiazolinone biocides, the agricultural fungicide chlorothalonil, and aliphatic amine epoxy catalysts will cause immediate burns on skin contact. Weaker agents, such as soap, detergents, solvents, and water, will slowly damage the epidermal barrier and cause dermatitis only after cumulative exposure. 
Fine or coarse particles of sand, sawdust, metal filings, or plastic may be blown on exposed surfaces and cause mechanical irritation. Tiny fibreglass needles penetrate deeply in the skin and create an intensely itchy dermatitis that mimics scabies. Plants have husks, thorns, and spines that produce foreign body granulomas. Other plants, such as dieffenbachias, philodendrons, agaves, and daffodils, contain high levels of oxalic acid responsible for the epidemic of dermatitis in gardeners and florists. Plants of the Apiaceae (eg, celery, carrot, parsnip, fennel) and Rutaceae (citrus fruits) families contain phototoxic psoralens. Skin contact with the sap or juice of these plants, followed by sunlight exposure, will cause an erythematous or bullous burn that heals with intense pigmentation. $^{5}$

\section{Allergens}

The most common occupational sensitizers are metal salts (Table 4). Hexavalent chromium is present in cement, corrosion-inhibiting primer paints, and coolants and is used to tan leather. Cobalt and nickel, the most common contact sensitizer, are ubiquitous in the metalworking industry. Mercury from amalgam is a hazard in the dental profession. Gold allergy, once thought to be rare, is now detected with increasing frequency among jewellers, dentists, and electronic technicians.

Rubber additives, such as mercaptobenzothiazoles, thiurams, carbamates, and thioureas, sensitize workers who manufacture rubber objects and those who use them, especially health personnel, housekeepers, or anyone who must wear rubber gloves for asepsis or protection. Phenolic, epoxy, or acrylate resins and their catalysts are also potent allergens that often sensitize assemblers in the aerospace and watercraft building industry, printers, dentists, and beauticians who apply artificial nails. Hairdressers are at risk from paraphenylenediamine in hair dyes, glyceryl thioglycolate in permanent-waving solutions, ammonium persulphate in bleach, and cocamidopropylbetaine in shampoos, among others. Allergenic biocides are found in metalworking fluids, paints, glues, water treatment additives, slimicide solutions in paper mills, hospital and housekeeping disinfectants, industrial soaps, and protective creams.

Numerous plants synthesize allergenic compounds. The sap of Toxicodendron radicans (poison ivy) contains urushiol, a mixture of penta- and heptadecylcatechols, extremely potent sensitizers responsible for $90 \%$ of cases of phytodermatitis in North America. Foresters and other outdoor workers are the primary victims of this severe
Table 4. Common Occupational Contact Allergens

Metals

Nickel, chromium, cobalt, mercury, gold, platinum

Rubber additives

Accelerators: mercaptobenzothiazole, carbamates, thiurams, thioureas

Antioxidants: $\mathrm{N}$-phenyl- $\mathrm{N}$-isopropyl-paraphenylenediamine, etc.

Plastics and resins

Epoxy, phenolic and acrylic monomers

Amine, anhydride, and peroxide catalysts

Colophony, turpentine, catechols

Biocides

Formaldehyde and formaldehyde releasers

Glutaraldehyde

Isothiazolinones

Methyldibromoglutaronitrile

Iodopropynyl butylcarbamate

Cosmetics

Paraphenylenediamine

Glyceryl thioglycolate

Cocamidopropylbetaine

Parabens and other preservatives (see biocides)

Fragrances and essential oils

Plants

Penta- and heptadecylcatehols

Sesquiterpene lactones

form of ACD. Farmers, gardeners, florists, and food handlers can at times become sensitized to sesquiterpene lactones in Asteraceae, Magnoliaceae, alstroemeria, and tulips. Sesquiterpene lactones are also present in bryophytes, such as Frullania dilatata, moss-like plants that grow on the bark of trees and cause seasonal dermatitis that forestry workers dub "cedar or wood poisoning." Plant-derived substances such as colophony, turpentine, essential oils, and fragrances are also notorious occupational allergens.

\section{Approach to Diagnosis and Management}

A diagnosis of occupational contact dermatitis can usually be suspected after a careful history and a thorough physical examination. Complementary testing will be required in most cases, and a visit to the workplace may occasionally be necessary, especially in the face of unexplained epidemics of contact dermatitis. Because it is easy to overlook important information during the initial consultation, Mathias proposed a series of seven objective criteria that form a framework for the correct identification of occupational contact dermatitis. ${ }^{6}$ If four of these 
criteria are present, the clinician can conclude that the dermatitis is probably of occupational origin (Table 5).

\section{History}

The date of onset of the dermatitis, its initial location, and its subsequent evolution should be ascertained. The physician must also note the effect of various treatments, holidays, and periods of sick leave. Important information also includes the name and address of the employer. The worker must state his or her job title and accurately describe the tasks performed. He or she should provide a list and Material Safety Data Sheets (MSDSs) of all products and chemicals handled, including cleansers and creams provided by the employer. The worker should also describe any protective equipment worn. Keeping in mind Mathias's criterion number 5, the physician should specifically ask about hobbies, personal habits, past history of skin disease, and use, outside the workplace, of cosmetics, protective moisturizers, and topical medicaments.

\section{Physical Examination}

When examining the affected areas, the physician will note the severity of the dermatitis, its distribution, and its degree of interference with function. He or she will also examine the entire integument as distant sites of involvement may harbour the telltale signs of atopic dermatitis, psoriasis, lichen planus, or another non-occupational, personal condition.

\section{Patch Testing}

A careful scrutiny of MSDSs will reveal exposure to irritants or allergens. The information that they contain is sometimes incomplete, but if the physician is confident that the affected worker has been exposed to irritants only, no further testing

Table 5. Mathias's Criteria of Occupational Causation of Contact Dermatitis

1. Clinical appearance consistent with contact dermatitis

2. Workplace exposure to potential cutaneous irritants or allergens

3. Anatomical distribution consistent with cutaneous exposure related to the job

4. Temporal relationship between exposure and onset consistent with contact dermatitis

5. Non-occupational exposures excluded as likely causes

6. Removal from exposure leads to improvement of dermatitis

7. Patch or provocation tests implicate a specific workplace exposure is necessary. If there is suspicion that the patient has been exposed to potential allergens, patch testing should be performed to confirm or rule out allergic sensitization. This in vivo bioassay is of undisputable value in the identification of the causative agents of ACD. It is easy to perform, but its difficulty lies in the interpretation of the results and the determination of their relevance to the worker's condition. Therefore, patch testing should be carried out by a physician who possesses a sound expertise in occupational problems and has access to a wide range of allergens.

Close to 400 standardized allergens are currently available from different suppliers (Table 6). Most are mixed in petrolatum or water and sold in individual syringes or vials. They are grouped by allergens in series, such as the rubber, metals, and glues and adhesives series, or by profession, such as the dental, hairdressers', or bakers' series. The TRUE Test is a prepackaged, ready to apply kit consisting of two adhesive panels in which the 23 allergens of the European standard series are embedded. Quick and easy to use, it must, however, often be supplemented by additional allergens as even the North American standard series, with 50 allergens, is insufficient to pick up all cases of occupational ACD. ${ }^{7}$

At the time of testing, the dermatitis should be in a quiescent phase and patients, ideally, should be off systemic corticosteroids or at least taking less than $20 \mathrm{mg}$

Table 6. List of Canadian Suppliers of Patch Test Materials

Dormer Laboratories Inc.

Distributor of Chemotechnique Diagnostics allergens and IQ

Chambers

Address: 91 Kelfield Street, \#5, Rexdale, ON M9W 5A3

Tel: 416-242-6167; Fax: 416-242-9487

Internet: www.dormer.ca; E-mail: info@dormer.ca

Omniderm Inc.

Distributor of Trolab-Hermal allergens and Finn Chambers

Address: 987 Séguin Street, Hudson, QC J0P 1H0

Tel: 450-458-0158; Fax: 450-458-7499

E-mail: omniderm@videotron.ca

Spexell Pharma

Distributor of TRUE Test ${ }^{\mathrm{TM}}$ allergen panels

Address: 2180 Meadowvale Blvd, Suite 200, Mississauga, ON L5N $5 \mathrm{~S} 3$

Tel: 866-571-7739; Fax: 866-572-7739

Internet: www.truetest.ca

SmartPractice Canada

Distributor of AllergEAZE allergens and chambers

2175 29th Street NE, Unit b90, Calgary, AB T1Y 7H8

Tel: 866-903-2671; Fax: 866-903-2672

Internet: www.allergeaze.com; E-mail: info@allergeaze.com 
of prednisone per day. The procedure requires three visits at intervals of 48 hours and is therefore most commonly performed Monday (day 0), Wednesday (day 2), and Friday (day 4). On day 0 , the allergens are applied to rows of aluminum or plastic chambers mounted on hypoallergenic porous tape and fixed to the patients' back. Patients are instructed to keep their back dry for the whole week and to avoid exercise and sweating. At day 2, the location of the panels is marked on the patients' back and the strips are removed. The reactions are noted at this time and again when the patients return at day 4 . Later readings may at times be necessary. Patch test reactions are graded according to standards established by the International Contact Dermatitis Research Group ${ }^{8}$ :

$0=$ No reaction

$?=$ Doubtful reaction: mild macular erythema

$+=$ Weak reaction: palpable erythema

$++=$ Strong reaction: erythema, papules, edema, vesicles

$+++=$ Extreme reaction: large, bullous, or ulcerated

IR = Irritant reaction: glazed erythema, burn-like erosion, pustules, edge effect

In general, irritant reactions occur early and fade quickly, whereas allergic reactions exhibit a crescendo pattern over many days. It is therefore not recommended to perform only one reading at day 2 as many true positive reactions may be missed and some irritant reactions will be called positive.

All patch test reactions must be assessed for relevance, whether past or present, pertinent to work or not. Relevance is definite when a test is positive with the substance or object containing the suspected allergen. It is considered probable if the substance identified by patch testing can be verified as present in the known skin contactant of the patient. The patient must be given clear and written instructions about all of his or her allergens, but only those relevant to work will be included in a workers' compensation report.

It is often necessary to test products from the workplace. However, a basic principle is to never test an unknown substance. Thus, it is important to carefully examine MSDSs to avoid testing caustic or toxic chemicals. Safer materials must be diluted down to non-irritant concentrations and mixed in the appropriate vehicle according to published guidelines. ${ }^{9}$ Ten to 20 control subjects should test negative to such non-standard allergens before they can be applied to the patient's back.

The basic patch testing technique must at times be modified. Readings will be performed after 20 or 30 minutes when contact urticaria is suspected, remembering, however, that prick testing remains the best diagnostic tool in cases of protein contact dermatitis. Photopatch testing, which requires four visits because the allergens must be exposed to 5 to 10 joules of ultraviolet $A$ at day 1 , is the technique of choice for the evaluation of suspected photoallergic contact dermatitis. It should be remembered, however, that most cases of photocontact dermatitis are caused by plants and are phototoxic and not photoallergic. Photopatch testing such plant products is therefore not indicated as the results would be positive in every subject so tested.

\section{Treatment}

Acute, oozy lesions are best treated with saline or Burow solution thin wet dressings that dry up the exudate, followed by application of potent corticosteroid creams or lotions. Extensive dermatitis will benefit from a short course of systemic corticosteroids, and sedative antihistamines will be used to quell pruritus. Chronic, fissured, and scaly dermatitis is treated with liberal use of emollients and midstrength to potent topical corticosteroids.

\section{Workers' Compensation}

Each province or country has its own workers' compensation board. Physicians must be familiar with the organization of the system in their jurisdiction and diligently fill out the forms that will allow workers to be adequately compensated. Strict avoidance of noxious irritants and allergens is of paramount importance, and patients must be withdrawn from work until all offenders are clearly identified. Return to modified tasks will be postponed until the skin lesions have completely disappeared, keeping in mind that full restoration of the epidermal barrier requires another 4 to 5 weeks after visible healing.

The extent of permanent physical impairment, such as persistence of residual skin lesions or the presence of cutaneous sensitizations, must be precisely determined. It is also necessary to clearly define functional limitations, such as "this worker should avoid direct and airborne cutaneous exposure to uncured epoxy monomer," etc.

\section{Conclusion}

The successful management of occupational contact dermatitis requires a dedicated physician with an inquisitive mind and meticulous investigator techniques. This physician not only must be able to recognize and treat skin diseases but should, in addition, possess solid notions of 
chemistry, physics, industrial processes, botany, and epidemiology. Moreover, he or she should be familiar with the legal aspects of workers' compensation boards and not be afraid of testifying in court.

\section{References}

1. Adams RA. Medicolegal aspects of occupational skin diseases. Dermatol Clin 1988;6:121-9.

2. Mathias CGT. Periodic synopsis. Occupational dermatoses. J Am Acad Dermatol 1988;19:1107-14.

3. Sasseville D. Occupational contact dermatitis. In: Stellman JM, editor. The ILO encyclopedia of occupational health and safety. 4th ed. Geneva: International Labor Office; 1998. p. 12.9-13.
4. Rustemeyer T, van Hoogstraten IMW, von Blomberg BME, Scheper RJ. Mechanisms in allergic contact dermatitis. In: Frosch PJ, Menné T, Lepoittevin JP, editors. Contact dermatitis. 4th ed. Berlin: Springer; 2006. p. 11-45.

5. Sasseville D. Phytodermatitis. J Cutan Med Surg 1999;3:263-9.

6. Mathias CGT. Contact dermatitis and workers' compensation: criteria for establishing occupational causation and aggravation. J Am Acad Dermatol 1989;20:842-8.

7. Pratt MD, Belsito DV, DeLeo VA, et al. North American Contact Dermatitis Group patch test results, 2001-2002 study period. Dermatitis 2004;15:1-8.

8. Wilkinson DS, Fregert OS, Magnusson B, et al. Terminology of contact dermatitis. Acta Derm Venereol 1970;50:287-92.

9. De Groot AC. Patch testing: test concentrations and vehicles for 3700 allergens. 2nd ed. Amsterdam: Elsevier; 1994. 